\title{
Technological disaster prevention - the case of Malaysia
}

\begin{abstract}
Purpose - The purpose of this paper is to provide some definition and foundation principles regarding disaster management. The paper also tests the Malaysian major hazard installations through the awareness and application of the Malaysian National Security Council (MNSC) directive 20 .

Design/methodology/approach - Questionnaire was circulated on 177 MHIs throughout Malaysia. Respondents of various demographic characteristics answered the questionnaires. It is believed that people of different age, sex, educational level, experience, and management levels are expected to have different perception and response to disaster management questions. A total of 65 completed questionnaires were answered.

Findings - The analysis of the disaster management questionnaire highlighted that more than half of the MHIs in Malaysia are multinational installations. The analysis revealed that 61 per cent of the Safety, Health and Environment Managers were aware of the MNSC directive 20 and 62 per cent said that the MNSC directive 20 is relevant to their facilities. The analysis further showed that 62 per cent of the respondents think the MNSC directive 20 is essential to their MHIs. However, 31 per cent of the respondents said that the emergency response plan (ERP) is used as an alternative to the MNSC directive 20. In the light of this, more than half of the MHIs are not local organizations; the Safety and Health Managers apparently are familiar with the safety guidelines of their parent organizations. Therefore, the authorities that are responsible for the enforcement of the MHIs' relevant regulations should be vigilant and follow up the MHIs to apply the relevant regulations, which suit the safety culture of Malaysia.

Originality/value - This paper presents an overview on technological disaster prevention. The paper also shows the results of testing of the Malaysian major hazard installations which are aware of the MNSC directive 20. The Malaysian experience can be beneficial.
\end{abstract}

Keyword: Hazards; Technology led strategy; Disasters; Malaysia 